\title{
The Effects of Age, Sex, and Genotype on Self-Report Drunkenness Following a Challenge Dose of Alcohol
}

\author{
M. C. Neale $^{1}$ and N. G. Martin ${ }^{2}$
}

Age is a potential source of variation that contributes to differences between, but not within, twin pairs. In most genetic analyses of twin data, linear and other functions of age are usually removed prior to model fitting. This correction is typically applied only within twin groups of the same sex and zygosity, and no heterogeneity test of age regressions is performed. Here we include age as a variable in the model-fitting procedure and allow for tests of heterogeneity of age regressions across sex and zygosity groups. The LISREL formulation of the approach is illustrated with data collected from Australian twins on subjective impressions of drunkenness following alcohol consumption. The results indicate significant negative covariation of impressions of drunkenness with age. The data support a simple model of additive genetic and unique environmental variation. No evidence was found for sex differences in genetic or environmental components of variation.

KEY WORDS: twins; alcohol; LISREL.

The theoretical work and data analysis described in this paper were made possible by NATO Grant 86/0823 and grants from the Belgian National Research Fund, the State University of Gent, and the Catholic University of Leuven. We are also grateful to Drs. R. Vlietinck and R. Derom for excellent organization of the successful workshop. Data collection was made possible by a grant from the Australian Associated Brewers to N.G.M. and Drs. J. G. Oakeshott, J. B. Gibson, and G. A. Starmer and by grants from the Australian National Health and Medical Research Council. The authors were supported by NIH Grants MH40828 and AA-06781.

${ }^{1}$ Department of Human Genetics, Medical College of Virginia, Box 33, Richmond, Virginia 23298.

2 Queensland Institute of Medical Research, Bramston Terrace, Herston, Brisbane, Queensland, Australia 4006. 


\section{INTRODUCTION}

There are two aims of this paper. The first is to describe a general method of age correction for the analysis of twin data. The second aim is to increase understanding about the effects of age, sex, genotype, and the environment on subjective impressions of drunkenness. In many human behavior genetic studies, subjects range in age from young adults to the elderly. If a relationship exists between age and the variable under study, the results of fitting genetic models to data from which the effects of age have not been removed may be misleading. In the classical twin study, any regression of the phenotype on age generates increased between-pair variation, while the variance within pairs is unaffected. The net effect would be to increase both monozygotic (MZ) and (DZ) dizygotic twin correlations and, therefore, to inflate estimates of common environmental variation. Methods to correct for this bias have been in use for many years (e.g., Eaves et al., 1978). McGue and Bouchard (1984) have compared a number of different procedures that attempt to correct for the effects of age prior to model-fitting analyses. One approach is to treat each phenotypic score as independent and to calculate the residual score after regression on age. However, in the case of twins and other family relatives this method is clearly inappropriate, as the scores of the relatives are not independent for most variables. This problem may be avoided by computing partial correlation matrices with the effects of age removed. But while this may be readily achieved using statistical packages such as SPSS-X (1983) or SAS (1985), the age regression is normally calculated within groups. To compute the age regression regardless of group and to calculate covariance matrices from the residuals is more complicated and rarely seen in the literature. Even less common are tests for homogeneity of age regressions across sex and zygosity groups, which may be of considerable interest. In this paper we describe how age and other covariates may be treated at the model-fitting stage and illustrate the procedure with a LISREL model (Jöreskog and Sörbom, 1986) applied to data on subjective impressions of drunkenness.

\section{UNIVARIATE MODEL}

The rationale behind correction of twin data for the effects of a covariate such as age is very simple. Any variation associated with age contributes to between-pair variation but not to within-pair variance since twins are always of the same age. As a result, any linear effects of age on the variable under study will cause an inflation of estimates of common environmental variation. Often, variation caused by age is not of primary 
interest, and therefore a variety of methods of correction for the effects of age has been proposed. Here we suggest that age be incorporated in the linear model specified by LISREL. This approach has great convenience and allows for tests of a variety of hypotheses concerning the equality of age regressions across sex and zygosity groups. As a general approach, some care is required since maximum-likelihood model-fitting methods assume multivariate normality of all variables, and this should clearly apply to the age distribution of the sample. For samples with markedly nonnormal age distributions, the user may consider classifying subjects into a number of age groups, from which polyserial correlations may be estimated. LISREL VII is expected to be released soon. The new software permits the calculation of asymptotically distribution-free bestGLS estimators (Browne, 1984). With these new methods, hypothesis testing will still be possible for nonnormally distributed variables, so difficulties with the age structure of samples will be overcome.

In order to estimate parameters that reflect the regression of the phenotype $(P)$ of an individual on age $(A)$, we may write a structural equation

$$
P=a A+e R,
$$

where $R$ is the residual variation not associated with age. When we have genetically informative data, it is possible to partition the residual variation in the model, so that in the case of twins for example, we may write

$$
P_{T 1}=a A+h G_{T 1}+d D_{T 1}+e E_{T 1},
$$

where $P_{T 1}, G_{T 1}, d D_{T 1}$, and $E_{T 1}$ are the phenotype, additive genotype, dominant genetic, and specific environment of the first twin of a pair. A corresponding equation may be written for the second twin, and the model may be represented as a path diagram (Fig. 1). The data matrices for the analysis are therefore of the order $2 n+1$, where $n$ is the number of variables under analysis. In order to allow flexibility for possible changes to the model (Health et al., 1989), we specify ultimate variables as $\xi$ variables and the observed variables as $\eta$ variables. The matrix $\Lambda_{Y}$ is an identity matrix. The $\Gamma$ and $\Phi$ matrices for a LISREL model of the univariate case are as follows:

$$
\Gamma=\begin{gathered}
\text { Age } \\
P T_{1} \\
P T_{2}
\end{gathered}\left[\begin{array}{rrrrrrr}
v & 0 & 0 & 0 & 0 & 0 & 0 \\
a_{1} & h & 0 & d & 0 & e & 0 \\
a_{2} & 0 & h & 0 & d & 0 & e
\end{array}\right]
$$




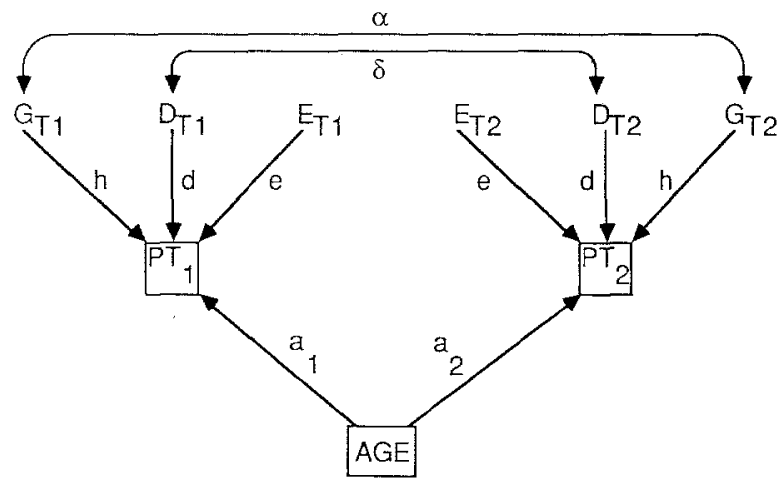

Fig. 1. Path diagram of a model of random environment $(E)$, additive genetic $(G)$, and either dominant genetic or common environmental $(D)$ variation in twins. The linear effects of age on the phenotypes $\left(P T_{i}\right)$ are effectively removed from the variation parameters by the explicit specification of age in the model. Parameters $a_{1}$ and $a_{2}$ (often constrained to be equal) are the regressions of age on the phenotypes.

$$
\begin{gathered}
\text { Age } G T 1 \\
\text { Age } \\
G T 12 \\
G T 2 \\
G T 2 \\
D T 1 \\
D T 2 \\
E T 1 \\
E T 2
\end{gathered}\left[\begin{array}{lllllll}
1 & 0 & 0 & 0 & 0 & 0 & 0 \\
0 & 1 & \alpha & 0 & 0 & 0 & 0 \\
0 & \alpha & 1 & 0 & 0 & 0 & 0 \\
0 & 0 & 0 & 1 & \delta & 0 & 0 \\
0 & 0 & 0 & \delta & 1 & 0 & 0 \\
0 & 0 & 0 & 0 & 0 & 1 & 0 \\
0 & 0 & 0 & 0 & 0 & 0 & 1
\end{array}\right]
$$

The parameter $\alpha$ is the correlation between the additive genetic components of twins, which is fixed at 1.0 for $\mathrm{MZ}$ and at .5 for $\mathrm{DZ}$ twins. The parameter $\delta$ can be used to specify either common environmental or dominant genetic variation. If $\delta$ is fixed at 1.0 for all twin groups, then a model including common environment is specified. Alternatively, if $\delta$ is fixed at 1.0 for monozygotic twins and at .25 for dizygotic twins, then a model including dominance variation is specified. Within LISREL, we have complete freedom to assign parameters for the age regressions both within and between groups. We may allow for different variances of age across groups or test for homogeneity of age variances by comparing the fit of this model with that of one where all age variances are constrained to be equal. It is possible to test for homogeneity of age regressions within groups, but with the exception of opposite-sex twin pairs, the test of equality is expected to be nonsignificant. The test likely to be of most interest is of the equality of age regressions between sexes. Clearly, we 
may extend this method to cover more complex models and multiple covariates. A simple example of multiple covariates is described by Vlietinck et al. (1989) in this issue.

Theoretically, common environmental variation may occur as a result of twins sharing certain characteristics, events, or experiences. Any linear effects of age on variation are part of the common environment, as age is perfectly correlated in twins. However, not all common environmental influences are necessarily unmeasured covariates which are perfectly correlated in this fashion. Age is an unusual covariate in this respect. Few other variables hold this feature, although certain aspects of the prenatal and rearing environment may also be identical for twins. We note that nonlinear effects of age may also be detected by including transformed age scores as covariates, although problems with nonnormality of the transformed scores would need to be addressed. The advent of LISREL VII should allow asymptotically distribution-free parameter estimation (Browne, 1984).

\section{SEX-LIMITATION MODEL}

The general approach to fitting a model of sex limitation to twin data is described by Heath et al. (1989). There are three general types of sex limitation in the causes of variation which are of interest. These are (i) "general scalar sex limitation" - a simple difference in total variance between males and females; (ii) "specific scalar sex limitation," where sex limitation is observed to different degrees for the genetic and environmental components of variance; and (iii) "nonscalar sex limitation," in which different genes or different environments are operating in the two sexes. The information to estimate effects $i$ and ii is available in the samesex twin pairs, but opposite-sex pairs are needed to estimate nonscalar effects.

In many cases, the approach described by Heath et al. is sufficient to define a model of scalar sex limitation. However, when opposite-sex twin pairs show a relatively low correlation, the absence of simple boundary constraints in LISREL becomes a problem in model fitting. In order to account for the low DZ opposite-sex correlation, LISREL will estimate negative genetic or common environmental paths in one sex but not the other. Such estimates can lower or even make the $\mathrm{DZ}$ covariance negative and effectively describe a model in which genetic (or environmental) effects correlate -1 between males and females. This specification is the opposite of that which is desired-genetic and environmental causes are assumed to correlate +1 between males and females. A respecification 
of the model is tedious but necessary to avoid this problem in LISREL VI.

A general approach to the specification of linear constraints is described by Rindskopf (1984). In order to specify constraints, intermediate dummy variables are added to the model, so that, for example, the correlation between two variables is $a^{2}$ rather than $a$. Since LISREL is gracious enough to constrain parameter estimates to real numbers, the estimate $a^{2}$ is always nonnegative. Rindskopf (1984) recommends the use of the $B$ matrix to specify constraints, and this approach is used here. Our aim is to have nonnegative estimates of additive genetic, common environment, and specific environment parameters. It is not necessary to constrain the specific environment parameters since these contribute only to variation within, not covariation between, the members of a twin pair, but this constraint is retained for the sake of consistency. We do not constrain the effects of age on the phenotype to be positive, as this may not be the case. In the unlikely event that the regression of age on the phenotype is positive in one sex and negative in the other, then indeed we would predict a lower correlation in DZ opposite-sex twins.

The full LISREL model for $y$ variables is required to implement the constraints. A diagram of the constrained model is shown in Fig. 2. Constraints are imposed in the usual way in LISREL with the EQ statements. Here we wish to impose constraints across matrices, such that equivalent elements of $\Gamma$ and $B$ are set equal. The matrix specifications are thus

$$
\begin{aligned}
& G_{1}{ }^{\prime} G_{2}{ }^{\prime} C_{1}{ }^{\prime} C_{2}{ }^{\prime} E_{1}{ }^{\prime} E_{2}{ }^{\prime} \text { Age1 Age2 Age' } P T 1 P T 2 \\
& \Lambda_{X}=\begin{array}{r}
P T 2 \\
\text { Age }
\end{array}\left[\begin{array}{lllllllllll}
0 & 0 & 0 & 0 & 0 & 0 & 0 & 0 & 1 & 0 & 0 \\
0 & 0 & 0 & 0 & 0 & 0 & 0 & 0 & 0 & 1 & 0 \\
0 & 0 & 0 & 0 & 0 & 0 & 0 & 0 & 0 & 0 & 1
\end{array}\right] \text {, } \\
& \begin{array}{c}
G_{1}{ }^{\prime} \\
G_{2}{ }^{\prime} \\
C_{1}{ }^{\prime} \\
C_{2}{ }^{\prime} \\
E_{1}{ }^{\prime} \\
E_{2}{ }^{\prime} \\
\text { Age1 } \\
\text { Age2 } \\
P T 1 \\
P T 2 \\
P T \\
\text { Age }^{\prime}
\end{array}\left[\begin{array}{rrrrrrrrrrr}
G_{1}{ }^{\prime} & G_{2}{ }^{\prime} & C_{1}{ }^{\prime} & C_{2}{ }^{\prime} & E_{1}{ }^{\prime} & E_{2}{ }^{\prime} \text { Age1 Age2 } & \text { Age } & P T 1 & P T 2 \\
0 & 0 & 0 & 0 & 0 & 0 & 0 & 0 & 0 & 0 & 0 \\
0 & 0 & 0 & 0 & 0 & 0 & 0 & 0 & 0 & 0 & 0 \\
0 & 0 & 0 & 0 & 0 & 0 & 0 & 0 & 0 & 0 & 0 \\
0 & 0 & 0 & 0 & 0 & 0 & 0 & 0 & 0 & 0 & 0 \\
0 & 0 & 0 & 0 & 0 & 0 & 0 & 0 & 0 & 0 & 0 \\
0 & 0 & 0 & 0 & 0 & 0 & 0 & 0 & 0 & 0 & 0 \\
0 & 0 & 0 & 0 & 0 & 0 & 0 & 0 & 0 & 0 & 0 \\
\sqrt{h} & 0 & \sqrt{c} & 0 & \sqrt{e} & 0 & 0 & 0 & 0 & 0 & 0 \\
0 & \sqrt{h} & 0 & \sqrt{c} & 0 & \sqrt{e} & 0 & a_{2} & 0 & 0 & 0
\end{array}\right],
\end{aligned}
$$




$$
\begin{gathered}
G_{1}^{\prime} \\
G_{2}{ }^{\prime} \\
C_{1}{ }^{\prime} \\
C_{2}{ }^{\prime} \\
E_{1}^{\prime} \\
E_{2}{ }^{\prime} \\
\text { Age1 } \\
\text { Age2 } \\
\text { Age' } \\
P T 1 \\
P T 2
\end{gathered}\left[\begin{array}{rrrrrrr}
G_{1} & G_{2} & C_{1} & C_{2} & E_{1} & E_{2} & \text { Age } \\
0 & 0 & 0 & 0 & 0 & 0 & 0 \\
0 & 0 & \sqrt{c} & 0 & 0 & 0 & 0 \\
0 & 0 & 0 & \sqrt{c} & 0 & 0 & 0 \\
0 & 0 & 0 & 0 & \sqrt{e} & 0 & 0 \\
0 & 0 & 0 & 0 & 0 & \sqrt{e} & 0 \\
0 & 0 & 0 & 0 & 0 & 0 & 1.0 \\
0 & 0 & 0 & 0 & 0 & 0 & 1.0 \\
0 & 0 & 0 & 0 & 0 & 0 & \sigma_{a} \\
0 & 0 & 0 & 0 & 0 & 0 & 0 \\
0 & 0 & 0 & 0 & 0 & 0 & 0
\end{array}\right],
$$

and

$$
\begin{array}{ccccccc}
\multicolumn{1}{c}{G T 1} & G T 2 & D T 1 & D T 2 & E T 1 & E T 2 & \text { Age } \\
G T 1 \\
G T 2 \\
D T 1 \\
D T 2 \\
E T 1 \\
E T 2 \\
\text { Age }
\end{array} \quad\left[\begin{array}{lllllll}
1 & \alpha & 0 & 0 & 0 & 0 & 0 \\
\alpha & 1 & 0 & 0 & 0 & 0 & 0 \\
0 & 0 & 1 & \delta & 0 & 0 & 0 \\
0 & 0 & \delta & 1 & 0 & 0 & 0 \\
0 & 0 & 0 & 0 & 1 & 0 & 0 \\
0 & 0 & 0 & 0 & 0 & 1 & 0 \\
0 & 0 & 0 & 0 & 0 & 0 & 1
\end{array}\right] .
$$

As in the earlier formulation in this paper, $\alpha$ is 1.0 for MZ twins and .5 for DZ twins. To specify a model of nonscalar sex-limitation (type iii above), $\alpha$ may be specified as a free parameter in the opposite-sex group. If $\delta$ is uniformly 1.0 , then a common environment effect is specified, or if it is fixed at .25 for $\mathrm{DZ}$ twins, then a model with genetic dominance is in effect. The estimates $\sqrt{h}, \sqrt{c}$, and $\sqrt{e}$, are the square roots of the parameter estimates obtained without constraints. In order to calculate the proportion of variation associated with any particular source, it is necessary to obtain the fourth power of the estimate and divide it by the total variation. The model may be specified more simply by use of the $\Gamma$ and $\Lambda_{Y}$ matrices but to do so would make the test of general scalar sex limitation difficult to specify. In addition, it is simple to specify a model of reciprocal phenotypic interaction within this model.

\section{SUBJECTS AND MEASURES}

The sex-limitation model was fitted to data collected by Martin $e t$ al. (1985) on the effects of an acute dose of alcohol on psychomotor and physiological responses. Twins were trained to plateau on apparatus mea- 
suring reaction time, coordination, and steadiness and measured while sober. They then drank a weight-related dose of alcohol $[0.75 \mathrm{~g} \mathrm{EtOH} /$ $\mathrm{kg}$ body weight, diluted to $10 \%(\mathrm{v} / \mathrm{v})$ in sugarless lemon squash] in 20 min. At 1,2, and $3 \mathrm{~h}$ after the start of drinking they were tested again on the psychomotor tasks. At these times we also asked the question, "How drunk are you now?" to which they responded on a 10-point scale from $1=$ "quite sober" to $10=$ "the most drunk I have ever been." Details of the subjects, measures, and protocol can be found elsewhere (Martin et al., 1985).

\section{RESULTS}

Means, standard deviations, and measures of skewness and kurtosis are shown in Table I for males and females. Variance-covariance and correlation matrices of age and self-report drunkenness for the two twins are shown in Table II. We recognize that the self-report drunkenness measures depart from multivariate normality. Although it would be possible to calculate polychoric and polyserial correlations from the data and, subsequently, to fit models to these statistics, this approach was rejected, as LISREL VI does not permit statistical tests of hypotheses with this type of data summary. Skewness or kurtosis will typically cause an inflation of $\chi^{2}$ for each model and submodel. This being the case, it is possible to reject a hypothesis that parameters are equal, when in fact they do not differ (type I error). However, if we find that estimates are not significantly different, then we may be fairly confident that this conclusion is correct. Hypotheses rejected at very high levels of significance are likely to remain rejected when distributional assumptions have been met.

A model including sex limitations seems a suitable starting point for data concerning alcohol, since sex differences are apparent for many aspects of alcohol use and abuse (e.g., Jardine and Martin, 1984). In view of the lack of power to estimate genetic dominance with small samples,

Table I. Univariate Statistics for Age and Self-Report Drunkenness (SRD) in Males and Females

\begin{tabular}{|c|c|c|c|c|c|c|c|c|}
\hline & \multicolumn{4}{|c|}{ Females $(N=213)$} & \multicolumn{4}{|c|}{ Males $(N=199)$} \\
\hline & Mean & $\mathrm{SD}$ & Skew & Kurt. & Mean & SD & Skew & Kurt. \\
\hline Age & 22.840 & 4.425 & .785 & -.346 & 23.271 & 4.840 & .623 & -.890 \\
\hline SRD & 6.545 & 2.591 & -.148 & -1.190 & 5.357 & 2.664 & .323 & -1.047 \\
\hline
\end{tabular}


Table II. Variance-Covariance (Diagonal and Above) and Correlation (Below Diagonal) Matrices for Age and a Subjective Index of Intoxication for Five Twin Groups

\begin{tabular}{|c|c|c|c|c|c|c|}
\hline & \multicolumn{3}{|c|}{ MZ females } & \multicolumn{3}{|c|}{$\mathrm{MZ}$ males } \\
\hline & Age & Twin 1 & Twin 2 & Age & Twin 1 & Twin 2 \\
\hline Age & 22.31 & -2.14 & .83 & 29.30 & -6.83 & -5.51 \\
\hline Twin 1 & -.18 & 6.60 & 3.50 & -.48 & 6.94 & 3.72 \\
\hline \multirow[t]{3}{*}{ Twin 2} & .06 & .48 & 8.14 & -.35 & .48 & 8.55 \\
\hline & \multicolumn{3}{|c|}{$\mathrm{DZ}$ females } & \multicolumn{3}{|c|}{$\mathrm{DZ}$ males } \\
\hline & Age & Twin 1 & Twin 2 & Age & Twin 1 & Twin 2 \\
\hline Age & 16.96 & -.78 & .17 & 15.07 & -3.29 & -1.91 \\
\hline Twin 1 & -.08 & 5.22 & -.55 & -.31 & 7.63 & 1.01 \\
\hline \multirow[t]{6}{*}{ Twin 2} & -.10 & -.09 & 7.74 & -.20 & .15 & 5.94 \\
\hline & \multicolumn{6}{|c|}{ DZ opposite sex } \\
\hline & & \multicolumn{2}{|c|}{ Age } & \multicolumn{2}{|c|}{ Twin 2} & \\
\hline & \multirow{2}{*}{\multicolumn{2}{|c|}{$\begin{array}{l}\text { Age } \\
\text { Twin } 1\end{array}$}} & & \multicolumn{2}{|c|}{-.04} & \\
\hline & & & & \multirow{2}{*}{\multicolumn{2}{|c|}{$\begin{array}{r}.45 \\
6.31\end{array}$}} & \\
\hline & \multicolumn{2}{|c|}{ Twin 2} & & & & \\
\hline
\end{tabular}

the sex-limitation additive genetic, common environment model (Fig. 2) was fitted by maximum likelihood (Neale et al., 1989) to the data in Table II. Parameter estimates and $\chi^{2}$ goodness-of-fit statistics for this scalar sex-limitation model and a variety of submodels are shown in Table III. The initial model fits the data very well, despite the possible departures from normality. Since the nonscalar sex-limitation model fits well, no examination of the nonscalar sex-limitation model is necessary.

Submodels II, III, and IV test a variety of hypotheses about age effects. The likelihood-ratio test is used to assess the significance of parameter estimates against model I. Submodel II indicates that the regression of age on the self-report does not differ between the sexes, as the difference $\chi^{2}$ of $(21.00-17.34)=3.66$ has 1 degree of freedom and is nonsignificant. Submodel III shows that the regression of age on selfreport drunkenness is highly significant (difference $\chi^{2}=11.82$; $\mathrm{df}=2$; $p<.001$ ). There is no evidence for heterogeneity of age variance between the five groups, as shown by submodel IV. This model was retained as a model against which differences in causes of phenotypic variance were tested. Model $\mathrm{V}$ indicates that there is no evidence of sex differences in genetic, common environment, or specific environmental variation. Re- 


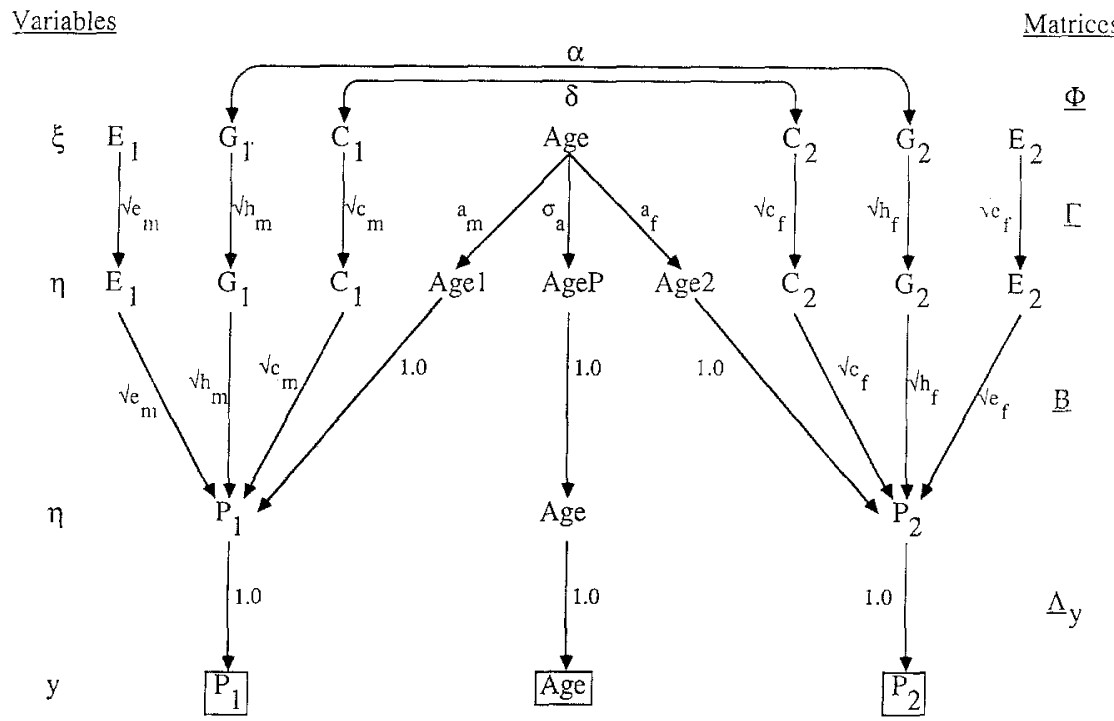

Fig. 2. Path diagram for scalar sex limitation in a pair of opposite-sex DZ twins. The LISREL specification is shown in terms of variables and matrices in the left- and right-hand columns. Observed variables are shown in boxes; subscripts $m$ and $f$ refer to male and female, respectively. Parameters $h, c$, and $e$ are constrained to be positive by specifying dummy variables in the model. Age is incorported as a covariate, such that $\sigma_{a}{ }^{2}$ is the variance of age, and $a_{m}$ and $a_{f}$ are the regressions of age on the phenotypes of male and female twins, respectively.

taining a model without sex differences, models VI, VII, and VIII show that while there is no evidence of common environmental variation, there is support for additive gene action as a source of variation in self-report drunkenness.

\section{DISCUSSION}

Incorporation of age into model-fitting analysis of individual differences has several advantages over conventional methods. First, the statistical treatment is correct, given that distributional assumptions have been met. Second, tests of significance of age effects are simple to perform. Third, a variety of tests may be made, including (i) difference of regressions from zero; (ii) group differences of variance in age; (iii) group differences in age regressions; and (iv) differences in age regressions between variables, especially repeated measures. "Group" may be classified by zygosity or sex or both. In the event that age is not normally distributed, it is still theoretically possible to test for the linear effects of 
Table III. Parameter Estimates and Goodness-of-Fit Statistics Obtained from Fitting a Variety of Submodels of the Path Model in Fig. 2 to the Data in Table II

\begin{tabular}{lrrrrrrrr}
\hline & \multicolumn{7}{c}{ Model } \\
\cline { 2 - 10 } Parameter & \multicolumn{1}{c}{ I } & \multicolumn{1}{c}{ II } & \multicolumn{1}{c}{ III } & \multicolumn{1}{c}{ IV } & \multicolumn{1}{c}{ V } & VI & VII & VIII \\
\hline$h_{m}$ & 1.52 & 1.58 & 1.75 & 1.56 & 1.55 & - & 1.55 & - \\
$h_{f}$ & 1.51 & 1.54 & 1.52 & 1.55 & 1.55 & - & 1.55 & - \\
$c_{m}$ & .00 & .00 & .00 & .00 & .00 & 1.20 & - & - \\
$c_{f}$ & .00 & .00 & .00 & .00 & .00 & 1.20 & - & - \\
$e_{m}$ & 2.06 & 2.04 & 2.01 & 2.04 & 2.06 & 2.28 & 2.06 & 2.58 \\
$e_{f}$ & 2.07 & 2.07 & 2.08 & 2.07 & 2.06 & 2.28 & 2.06 & 2.58 \\
$a_{m}$ & -.70 & -.43 & - & -.45 & -.45 & -.46 & -.45 & -.46 \\
$a_{f}$ & -.17 & -.43 & - & -.45 & -.45 & -.46 & -.45 & -.46 \\
$\sigma_{A 1}$ & 4.73 & 4.79 & 4.72 & 4.55 & 4.55 & 4.55 & 4.55 & 4.55 \\
$\sigma_{A 2}$ & 5.24 & 5.24 & 5.41 & 4.55 & 4.55 & 4.55 & 4.55 & 4.55 \\
$\sigma_{A 3}$ & 4.11 & 4.16 & 4.12 & 4.55 & 4.55 & 4.55 & 4.55 & 4.55 \\
$\sigma_{A 4}$ & 3.89 & 3.83 & 3.88 & 4.55 & 4.55 & 4.55 & 4.55 & 4.55 \\
$\sigma_{A 5}$ & 4.55 & 4.49 & 4.39 & 4.55 & 4.55 & 4.55 & 4.55 & 4.55 \\
$\chi^{2}$ & 17.34 & 21.00 & 29.16 & 25.79 & 25.80 & 31.86 & 25.80 & 41.50 \\
$\mathrm{df}$ & 17 & 18 & 19 & 22 & 25 & 26 & 26 & 27 \\
$p$ & .43 & .28 & .06 & .26 & .42 & .20 & .47 & .04 \\
\hline
\end{tabular}

age by defining a discrete number of classes of age and computing polyserial or polychoric correlations with the variables of interest. It is also possible to test for nonlinear effects of age by fitting to, e.g., the square of age. Although it is possible to consider other covariates in this fashion, caution should be taken not to treat variables as covariates unless the twin correlation on the variable is perfect in every group. For variables on which the members of a twin pair differ, traditional multivariate analyses are to be preferred.

A model including age and sex limitation was fitted to the data on self-report drunkenness. Of the eight models fitted to the data, model VII, involving equal age variances and regressions across groups and simple additive genetic and common environmental variation, is selected as a parsimonious account of the data. Parameter estimates indicate approximately $35 \%$ of the phenotypic variation in SRD to be associated with additive genetic effects, with no effect of the common environment. There is a moderate negative regression of age with self-report drunkenness, indicating that older persons report less drunkenness than younger persons. This effect may arise from higher levels of alcohol tolerance acquired by older persons, but the implication is that the older person is more likely to ignore the effects of alcohol on any decision-making process. No evidence for sex differences in variation is found for this occasion 
of measurement, although inspection of the data for subsequent occasions of measurement suggests that this may not be the case in general.

There is no evidence for the effects of rearing or family environmental effects shared by twins on subjective impressions of drunkenness. This absence of common environmental effects is often found for measures of personality, and there may be substantial effects of personality variables on the self-report of drunkenness. However, it seems likely that subjective impressions of drunkenness under a challenge dose of alcohol are determined at least in part by previous drinking experience. The net effects of sociocultural variables such as educational, occupational, and marital status and urban or rural habitat, known to be associated with alcohol use and abuse (Riley and Marden, 1947; Mulford, 1964; Cahalan and Room, 1974; Weissman et al., 1980; Clark and Midanik, 1982), might be expected to show some effect of the common environment in $\mathrm{SRD}$, but this is not apparent in our data. The sample is small, and while the design is more powerful, in principle, for the detection of common environmental than for genetic effects (Martin et al., 1978), it is possible that sample truncation has obscured any effects of the shared environment (Neale et al., 1989).

In this issue, Martin and Boomsma (1989) show genetic variance for willingness to drive when drunk and that much of this genetic variance is shared by the personality trait extraversion. Common environment for willingness to drive is important only on the first occasion. In addition, the relationships between subjective ratings of drunkenness and objective measures of psychomotor performance or blood alcohol concentrations would be of considerable interest.

Evidence for additive genetic variation in SRD is no surprise. In humans, there is a substantial body of evidence for genetic variation in alcohol consumption (e.g., Partanen et al., 1966; Cederlöf et al., 1977; Kaprio et al., 1981, 1987; Clifford et al., 1984; Jardine and Martin, 1984; Heath et al., 1988; Heath and Martin, 1988). The consistent findings for human alcohol use are supported by research on selective breeding of mice and rats, which indicates genetic influences on ethanol consumption, initial central nervous system sensitivity, and the ability to acquire tolerance (McClearn and Erwin, 1982). There may be a close causal or interactive relationship between SRD and these phenotypes in humans. It seems likely that genetic influences on subjective impressions of drunkenness share a large proportion in common with genetic variation in drinking behavior. Large and comprehensive twin studies are needed to test hypotheses about the genetic and environmental causes of the relationships between these phenotypes. 


\section{APPENDIXES I AND II}

\section{Appendix I}

LISREI input file to fit a simple univariate molet of uwin data (shown in Figure 1), allowing for age as a covariate. The data matrices are of order $(\bar{r}, \bar{i})$ from which 3 variables are selected. The Mo card for the first group has been split over two lines; cuncatenation of these lines is necessary for LISREL input.

"HOW DRUNK" T1 - T3 - M7,

DA $\mathrm{NG}=2 \mathrm{NI}=7 \quad \mathrm{NO}=43$

LA

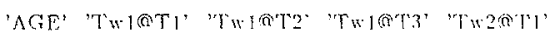
'Гw2@'Г2' 'Tw2@T3'

CM FI=MZDRUNK.COV

SE

$125 /$

MO NY=3 $\quad \mathrm{NX}=0 \quad \mathrm{NE}: 3 \quad \mathrm{NK}=7 \quad$ GA $\quad \mathrm{FU}, \mathrm{FI}$

PH $=S Y, F I$ LY $=$ ID PS: $Z \mathrm{ZE}$ 'TE, $\cdots \mathrm{ZE}$,

LE

'AGE' 'P1' 'P2'

LK

*

'ACE' 'G1' 'G2' 'D1' 'D2' 'E1' 'E2'

PA GA

*

1000000

110101010

1010101

$\mathrm{EQ} \mathrm{GA}(2,2)$ G $\Lambda(3,3)$

$\mathrm{EQ} \mathrm{GA}(2,4) \mathrm{GA}(3,5)$

EQ GA(2,6) GA(3,7)

EQ GA $(2,1)$ GA $(3,1)$

MA PH

1.

0. 1 .

0. I. 1.

0. 0. 0. 1

0. 0.0.1.1.

0. 0.0 .0 .0 .1 .

0. $0.0 .0 .0 .0 .1 . /$

ST. 5.0 ALL

OU SE

HOW DRUNK? T1 - T' $3-\mathrm{DZ}$

DA $\mathrm{NO}=42$

LA

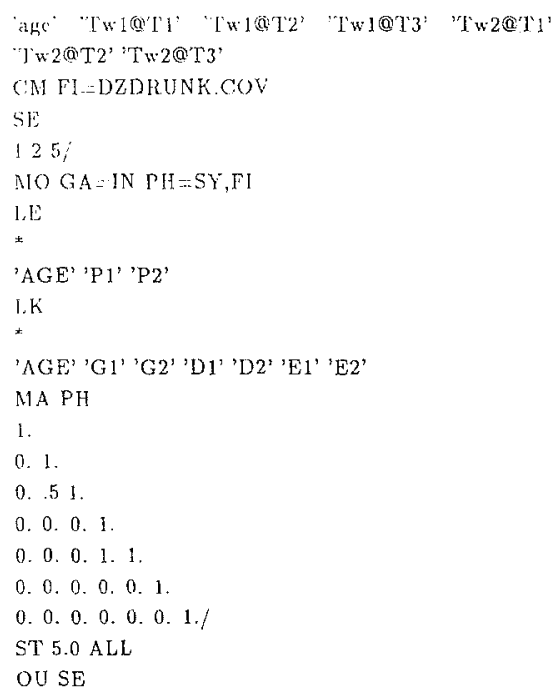

\section{Appendix II}

Start of LISREL input file to fit the model shown in Figure 2. The datia matrices are of order $(7 \times 7)$ from which 3 variables are selected. The MO card for the first group has been split over two lines; concatenation of these lines is necessary for LISREL input.

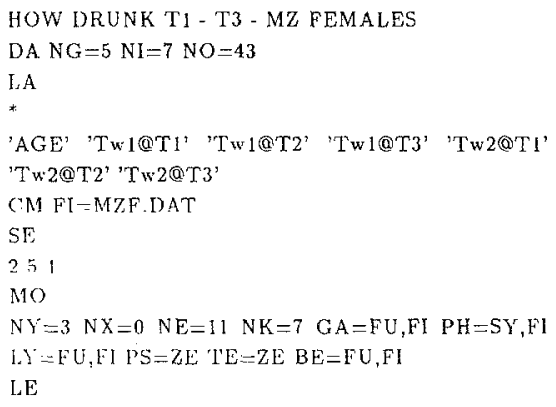

Fig. A1 


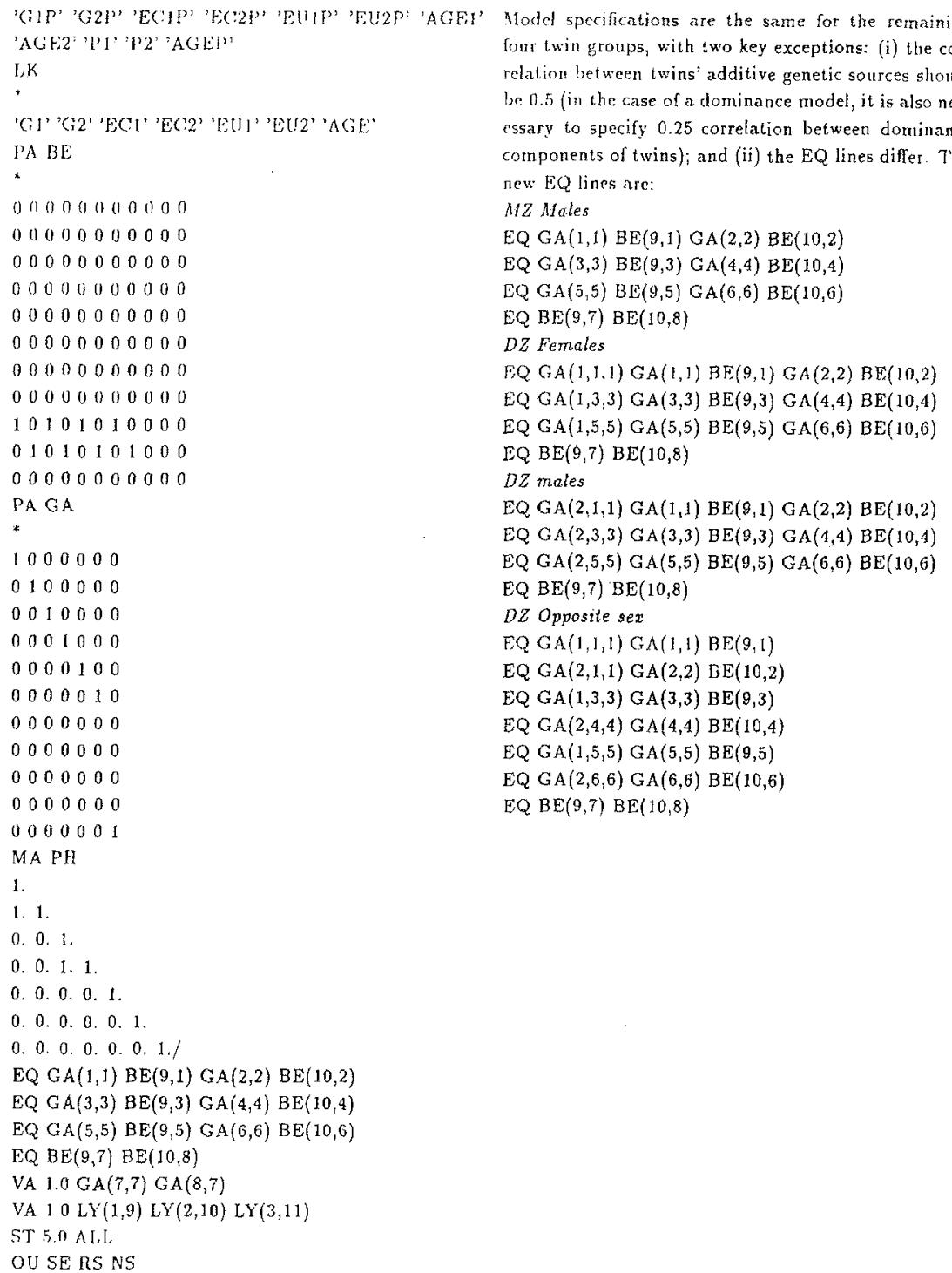

Fig. A1 (Continued) 


\section{REFERENCES}

Browne, M. W. (1984). Asymptotically distribution free methods for the analysis of covariance structures. Br. J. Math. Stat. Psychol. 37:62-83.

Cahalan, D., and Room, R. (1974). Problem Drinking Among American Men, Center of Alcohol Studies Monograph No. 6, New Brunswick, N.J.

Cederlöf, R., Friberg, L., and Lundman, T. (1977). The interactions of smoking, environment and heredity and their implications for disease aetiology. Acta Med. Scand. 202(Suppl. $612): 1-128$.

Clark, W. B., and Midanik, L. (1982). Alcohol use and alcohol problems among U.S. adults: Results of the 1979 national survey. In NIAAA (eds.), Alcohol Consumption and Related Problems, USGPO, Washington, D.C.

Clifford, C. A., Hopper, J. L., Fulker, D. W., and Murray, R. M. (1984). A genetic and environmental analysis of a twin family study of alcohol use, anxiety and depression. Genet. Epidemiol. 1:63-79.

Eaves, L. J., Last, K., Young, P. A., and Martin, N. G. (1978). Model fitting approaches to the analysis of human behaviour. Heredity $41: 249-320$.

Heath, A. C., and Martin, N. G. (1988). Teenage alcohol use in the Australian twin register: Genetic and social determinants of starting to drink. Alcohol Clin. Exp. Res. (in press).

Heath, A. C., Jardine, R., and Martin, N. G. (1988). Interactive effects of genotype and social environment on female alcohol consumption. J. Stud. Alcohol (in press).

Heath, A. C., Neale, M. C., Hewitt, J. K., Eaves, L. J., and Fulker, D. W. (1989). Testing structural equation models for twin data using LISREL. Behav. Genet. 19:9-35.

Jardine, R., and Martin, N. G. (1984). Cause of variation in drinking habits in a large twin sample. Acta Genet. Med. Gemellol, 33:435-450.

Jöreskog, K. G., and Sörbom, D. (1986). LISREL: Analysis of Linear Structural Relationships by the Method of Maximum Likelihood, National Educational Resources, Chicago.

Kaprio, J., Koskenvuo, M. D., and Sarna, S. (1981). Cigarette smoking, use of alcohol and leisure time physical activity in same sexed adult twin pairs. In Twin Research 3: Epidemiology \& Clinical Studies, A. R. Liss, New York, pp. 37-46.

Kaprio, J., Koskenvuo, M. D., Langinvainio, H., Romanov, K., Sarna, S., and Rose, R. J. (1987). Genetic influences on use and abuse of alcohol: A study of 5638 adult Finnish brothers. Alcohol Clin. Exp. Res. 11:349-356.

Martin, N. G., and Boomsma, D. (1989). Willingness to drive when drunk and personality: A twin study. Behav. Genet. 19:97-111.

Martin, N. G., Eaves, L. J., Kearsey, M. J., and Davies, P. (1978). The power of the classical twin study. Heredity 40:97-116.

Martin, N. G., Oakeshott, J. G., Gibson, J. B., Starmer, G. A., Perl, J., and Wilks, A. V. (1985). A twin study of psychomotor and physiological response to an acute dose of alcohol. Behav. Genet. 15:305-347.

McClearn, G., and Erwin, V. (1982). Mechanisms of genetic influence on alcohol related behaviors. In NIAAA (eds.), Alcohol Consumption and Related Problems, USGPO, Washington, D.C.

McGue, M., and Bouchard, T. (1984). Adjustment of twin data for the effects of age and sex. Behav. Genet. 18:325-343.

Mulford, H. A. (1964). Drinking and deviant drinking, USA, 1963. Q. J. Stud. Alcohol 25:634-650.

Neale, M. C., Eaves, L. J., Kendler, K. S., and Hewitt, J. K. (1989). Bias in correlations from selected samples of relatives: The effects of soft selection. Behav. Genet. 19:(in press).

Partanen, J., Bruun, K., and Markkanen, T. (1966). Inheritance of Drinking Behavior: A Study of Intelligence, Personality and Use of Alcohol of Adult Twins, Finnish Foundation for Alcohol Studies, Helsinki.

Riley, J. W., and Marden, C. F. (1947). The social pattern of alcoholic drinking. Q. J. Stud. Alcohol 8:265-273. 
Rindskopf, M. (1984). The use of phantom and imaginary latent variables to parameterize constraints in linear structural models Psychometrika 49:37-47.

SAS (1985). SAS User's Guide: Basics, Version 5 Edition, SAS Institute Inc., Cary, N.C. SPSS (1983). SPSS X User's Guide, McGraw-Hill, London.

Vlietinck, R., Derom, R., Neale, M. C., Maes, H. van Loon, H., van Maele, G., Derom, C., and Thiery, M. (1988). Genetic and environmental variation in the birth weight of twins. Behav. Genet. 19:151-161.

Weissman, M. M., Meyers, K., and Harding, P. (1980). Prevalence and psychiatric heterogeneity in a United States urban community. J. Stud. Alcohol 41:672-681. 